\title{
Our Hybrid Approach in the Treatment of Peripheral Vascular Diseases
}

\author{
(1) Cihan Yücel, (D) Mete Gürsoy, (D) Serkan Ketenciler, (D) Burçin Çayhan Karademir, (D) Nihan Kayalar \\ University of Health Sciences Turkey, Prof. Dr. Cemil Taş̧̧ığlu City Hospital, Clinic of Cardiovascular Surgery, Istanbul, Turkey
}

\section{Abstract}

Objective: Peripheral arterial disease is a critical health problem with an incidence of approximately $13 \%$ in the population over 50 years of age. Although endovascular interventions provide successful results as sole treatment in many patients, concomitant surgical interventions may be necessary to provide complete revascularization of the target extremities in complex peripheral vascular disease cases. In this study, we aimed to present the early results of 21 patients who underwent hybrid peripheral vascular intervention

Methods: Twenty one patients who underwent hybrid procedures for peripheral arterial disease between January 2018 and June 2018 were included in the study. One of the patients underwent an open surgery primarily, and the endovascular intervention was applied after 1 month of surgery. Endovascular and surgical procedures were performed simultaneously in all other patients.

Results: Fifteen patients were male (71.4\%), and 6 were female (28.6\%). The mean age of patients was $61.95 \pm 7.88$ [mean \pm standard deviation/ standard error of mean (SD/SEM)] years, with an age range of 52-75 years. The average ankle-brachial index of the targeted extremity was $0.25 \pm 0.12$ (mean \pm SD/SEM).

Conclusion: The hybrid procedures provided the best benefit possible for the complex arterial lesions with increased inflow and distal flow. Keywords: Hybrid method, peripheral vascular diseases, endovascular treatment

\section{INTRODUCTION}

Peripheral arterial disease is an important health problem with an incidence of approximately 13\% in the population over 50 years of age (1). Although there are many etiological factors, atherosclerosis is the most common cause (2). Because of the systemic nature of atherosclerosis, $60 \%$ of these patients have simultaneous coronary artery disease, and 30\% have cerebrovascular pathologies (3). 10-15\% of the patients with intermittent claudication die of cardiovascular diseases within 5 years. Although many patients may be asymptomatic, the indication of the intervention and the clinical classification are determined according to the severity of the symptoms. Claudication is the main reason for intervention in most patients, and the type of intervention depends on the severity of the disease.
In the last two decades, due to the rapidly improving endovascular intervention methods and increasing experience in this field, catheter-based procedures have become the first line of treatment in peripheral artery disease (PAD). Therefore, this approach is considered the "Endo-first" approach. Although endovascular interventions provide successful results as sole treatment in many patients, concomitant surgical interventions may be necessary to provide complete revascularization of the target extremities in complex peripheral vascular disease cases. Although hybrid operating room settings provide ideal conditions, the C-arm scope device in standard operating rooms can be sufficient in many cases. This study aimed to present the early results of 21 patients who underwent hybrid peripheral vascular surgery with the help of a C-arm scope device under standard operating room conditions between January-June 2018 in our clinic. 


\section{METHODS}

\section{Patient Selection}

In this study, data from the clinical database were analyzed retrospectively. The patients admitted to the cardiovascular surgery clinic and diagnosed with PAD and then underwent hybrid intervention were analyzed.

The ankle-brachial index (ABI) of all patients was measured after a detailed history and physical examination. In patients with $\mathrm{ABI}$ $<0.9$, computed tomographic (CT) angiography was performed. In addition, all patients underwent carotid doppler and cardiac examination, except for two patients who were operated on under emergency conditions. Patients were classified by using Fontaine classification, and patients with Fontaine 2B or higher with comorbidities were considered and treated with hybrid therapy. All patients participating in the study were informed about the study, and their consent was obtained. Ethics Committee Okmeydanı Training and Research Hospital approved this study (approval number: 48670771-514.10, date: 14.05.2019).

\section{Surgical Technique}

Operations were performed under either general anesthesia, local anesthesia, or peripheral nerve block. Long superficial femoral artery (SFA) occlusions $(>25 \mathrm{~cm})$ were treated with prosthetic bypass grafting in elective conditions. Endarterectomy was performed for the lesions, which may cause anatomic and physiological stenosis in the main femoral artery or to relieve stenoses limiting the inflow. In addition, simultaneous profundoplasty was performed in the presence of osteal stenosis of the deep femoral artery.

Endovascular treatment methods were applied to patients with aorta-iliac lesions shorter than $5 \mathrm{~cm}$, SFA lesions shorter than $25 \mathrm{~cm}$, or distal arterial lesions. Endovascular techniques used were atherectomy, percutaneous balloon angioplasty, and/ or drug-coated percutaneous balloon angioplasty. In addition, since most patients had simultaneous endovascular and surgical procedures, catheterization was performed from the explored artery for endovascular treatment.

\section{Statistical Analysis}

Statistical analyzes were performed using SPSS (SPSS Inc., Chicago, IL, USA) version 16.0. Continuous variables are presented as mean \pm standard deviation and categorical variables as $n$ and percentage values.

\section{RESULTS}

The study included a total of 21 patients who underwent hybrid procedures for peripheral arterial disease between January 2018 and June 2018. 15 of the patients were male (71.4\%), and 6 were female (28.6\%). The mean age of patients was $61.95 \pm 7.88$ years, with an age range of 52-75 years. The most common risk factor was smoking (80.9\%). The other risk factors related to the patients were summarized in Table 1. Two patients (9.5\%) had ischemic wounds (Fontaine class 4), 14 patients (66.6\%) had claudication (Fontaine class 2B), and 5 patients (23.9\%) had rest pain (Fontaine class 3 ). The average $\mathrm{ABI}$ in the targeted extremity was $0.25 \pm 0.12$.

Interventions were performed in 18 patients under general anesthesia, 2 patients under local anesthesia, and one patient with a peripheral nerve block. Femoro-popliteal bypass was performed in 10 patients due to long-segment superficial femoral artery lesions $(>25 \mathrm{~cm})$. Six patients underwent endarterectomy to the main femoral artery. Extra anatomic femoro-femoral bypass was performed in 2 patients. Interposition with autogenous vein, bilateral embolectomy, and extra anatomic axillo-femoral bypass was performed in each one patient. In the same session, 6 patients underwent self-expandable main iliac artery stent implantation, 11 patients underwent anterior tibial artery percutaneous transluminal balloon angioplasty, and 3 patients underwent superficial femoral artery percutaneous transluminal balloon angioplasty. Detailed data on patient procedures are summarized in Table 2 .

For the patient who underwent femoro-femoral bypass and the deep femoral artery plasty, transposition from the superficial femoral artery to the deep femoral artery was performed percutaneous transluminal balloon angioplasty. An application of vacuum assisted closure for prolonged service hospitalization and wound infection was required in the inguinal incision in this patient. As a result, this patient increased the average length of

\begin{tabular}{|l|l|l|}
\hline \multicolumn{4}{|l|}{ Table 1. Demographic data } \\
\hline Risk factor & Number $(\mathbf{n})$ & Percentage (\%) \\
\hline Hypertension & 9 & 42.8 \\
\hline Diabetes mellitus & 13 & 61.9 \\
\hline Hyperlipidemia & 6 & 28.5 \\
\hline Coronary artery disease & 8 & 38 \\
\hline Smoking & 17 & 80.9 \\
\hline $\begin{array}{l}\text { Infrarenal abdominal aortic } \\
\text { aneurysm }\end{array}$ & 1 & 4.7 \\
\hline Carotid artery stenosis & 1 & 4.7 \\
\hline Small cell lung cancer & 1 & 4.7 \\
\hline
\end{tabular}




\begin{tabular}{|l|l|}
\hline \multicolumn{2}{|l|}{ Table 2. Surgical and endovenous procedures applied to the } \\
patients
\end{tabular}

stay. The mean length of hospitalization was $3.15 \pm 2.94$ days. There was no acute renal failure or mortality in the postoperative period. Two patients with ischemic injuries were referred to hyperbaric oxygen therapy at the end of postoperative follow-up. One of the two patients underwent interposition surgery with an autogenous graft to the popliteal artery under emergency conditions, and the other patient underwent bilateral femoral artery embolectomy operation. One of the patients underwent at first endovascular intervention before one month to open surgery. This patient underwent endovascular intervention guided by arterial doppler ultrasonography of the main femoral artery. The mean of AAI was $0.83 \pm 0.15$ in the postoperative follow-up period.

\section{DISCUSSION}

This retrospective study presents our experience in 21 patients who underwent a hybrid intervention. We performed simultaneous endovascular and surgical treatment in 20 patients and staged hybrid in one patient. The hybrid approach is defined as the combined utilization of open surgery and endovascular methods in the same case. PAD is a systemic disease. Ozkan et al. (4) reported their series of the 626 patients, 400 (64\%) had multisegmental disease, the most common form of combined femoropopliteal and crural disease (25\%). Neither open surgery nor endovascular approach could provide complete revascularization in some of these complex cases. The present study aimed to discuss the importance of the hybrid approach following current European Society for Vascular Surgery guidelines recommendations.

While the discussion on the choice of treatment continues, the latest published European Society of Cardiology guideline suggests endovascular treatment as the first choice for occlusive lesions shorter than $5 \mathrm{~cm}$ in terms of revascularization of aortailiac disease and aorta-bifemoral bypass class I defined as indicated for aorta-iliac occlusions without serious comorbidity. Furthermore, in patients with long and/or bilateral aortailiac occlusive lesions with severe comorbidity, the need for endovascular intervention was emphasized as the first strategy with class I indication.

In the same guideline, endovascular treatment for occlusive femoropopliteal lesions below $25 \mathrm{~cm}$ and autologous vein grafting for lesions above $25 \mathrm{~cm}$ are presented as the first strategy with class I indication. Transluminal balloon angioplasty and stenting process of the aorta-iliac region has a high primary and secondary patency rate. Primary patency in short lesions $(<5 \mathrm{~cm})$ after transluminal angioplasty is reported as $64.5 \%$, and secondary patency is found to be $81.8 \%$ (5).

The aortoiliac occlusive disease is generally associated with severe comorbidities. Aortobifemoral or aortobiiliac bypass is still the preferred approach in complex cases with 5 and 10 years patency rates of approximately $90 \%$ and $75 \%$, respectively $(6,7)$. Aortic bifurcated surgery is a high-risk procedure. Bredahl and colleagues reported 3.6\% of cases showed 30-day mortality, and $20 \%$ of cases showed 30-day major complications in their 20 years of experience (8). In the last decade, Endovascular Aneurysm Repair, Covered Endovascular Repair of Aortic Bifurcation (CERAB), and the kissing stent technique have become a current first line treatment in experienced centers. The endovascular approach has comparable midterm results but has better early-term outcomes regarding mortality, hospital stays, bleeding, infection, and mesenteric ischemia. We also prefer both CERAB and the kissing stent technique in complex cases. We reported 7 patients underwent simultaneous uniiliac endovascular intervention and peripheric arterial surgery with $100 \%$ procedural success in this series. Primary iliac stenting was the preferred technique in each case. Peripheric surgery was planned considering both preoperative CT angiography and DSA during the iliac procedure.

Jorshery et al. (9) compared hybrid interventions with a peripheral bypass with saphenous vein and prosthetic graft in patients with complex pad. They reported that hybrid procedures have favorable perioperative outcomes compared with open bypass for femoropopliteal revascularization. Our 
series combined femoropopliteal bypass and distal angioplasty in 7 cases, common femoral endarterectomy and angioplasty in 2 cases, and bilateral embolectomy and angioplasty in 1 case. Each case was discharged uneventfully with 100\% early-term success (9).

\section{CONCLUSION}

In conclusion, the hybrid procedures provide the best benefit possible for the complex arterial lesions with the increase of both inflow and distal run-off. Hybrid procedures may also reduce hospital stay, mortality, and morbidity.

\section{Ethics}

Ethics Committee Approval: Ethics Committee Okmeydanı Training and Research Hospital approved this study (approval number: 48670771-514.10, date: 14.05.2019).

Informed Consent: All patients participating in the study were informed about the study, and their consent was obtained.

Peer-review: Externally and internally peer-reviewed.

\section{Authorship Contributions}

Surgical and Medical Practices: C.Y., S.K., Concept: N.K., Design: N.K., Data Collection or Processing: C.Y., S.K., B.C..K., Analysis or Interpretation: M.G., Literature Search: M.G., B.C..K., Writing: C.Y.

Conflict of Interest: No conflict of interest was declared by the authors.

Financial Disclosure: The authors declared that this study received no financial support.

\section{REFERENCES}

1. Crawford F, Welch K, Andras A, Chappell FM. Ankle brachial index for the diagnosis of lower limb peripheral arterial disease. Cochrane Database Syst Rev 2016;9:CD010680.

2. Aronow WS. Management of peripheral arterial disease of the lower extremities in elderly patients. J Gerontol A Biol Sci Med Sci 2004;59:172-

3. Viles-Gonzalez JF, Fuster V, Badimon JJ. Atherothrombosis: a widespread disease with unpredictable and life-threatening consequences. Eur Heart J 2004;25:1197-207.

4. Ozkan U, Oguzkurt L, Tercan F. Atherosclerotic risk factors and segmental distribution in symptomatic peripheral artery disease. J Vasc Interv Radiol 2009;20:437-41

5. Abello N, Kretz B, Picquet J, Magnan PE, Hassen-Khodja R, Chevalier J, et al. Long-term results of stenting of the aortic bifurcation. Ann Vasc Surg 2012;26:521-6.

6. Lun Y, Zhang J, Wu X, Gang Q, Shen S, Jiang H, et al. Comparison of midterm outcomes between surgical treatment and endovascular reconstruction for chronic infrarenal aortoiliac occlusion. J Vasc Interv Radiol 2015;26:196-204.

7. Grimme FA, Goverde PC, Verbruggen PJ, Zeebregts CJ, Reijnen MM Editor's Choice--First Results of the Covered Endovascular Reconstruction of the Aortic Bifurcation (CERAB) Technique for Aortoiliac Occlusive Disease. Eur J Vasc Endovasc Surg 2015;50:638-47.

8. Bredahl K, Jensen LP, Schroeder TV, Sillesen H, Nielsen H, Eiberg JP. Mortality and complications after aortic bifurcated bypass procedures for chronic aortoiliac occlusive disease. J Vasc Surg 2015;62:75-82.

9. Jorshery SD, Skrip L, Sarac T, Ochoa Chaar Cl. Hybrid femoropopliteal procedures are associated with improved perioperative outcomes compared with bypass. J Vasc Surg 2018;68:1447-54. 